\title{
Sports Medicine and Applied Sport Science: Can we truly Bridge the Gap?
}

\section{David W. Keeley*}

Department of Human Performance, Dance, and Recreation, New Mexico State University, Las Cruces, USA

Sports medicine is a broad field with a focus on the prevention, evaluation, treatment, and rehabilitation of sports injuries. In recent years, the number of published studies in the Sports Medicine field has increased dramatically. This rise in publication rate may be attributed to two primary factors. The first factor functioning to increase research in Sports Medicine may be the worldwide growth in sport. As participation in sport has grown throughout the world, the number of Sports Medicine practitioners and the institutional support they receive have also increased. The second factor contributing to the increased volume of Sports Medicine research may be the current emphasis on providing the best possible medical and rehabilitation practices through the use of evidence based medicine (EBM).

Although both of these factors may have contributed to the increase in Sports Medicine research, much of the resulting research has been retrospective in nature. As a result of this retrospective approach, the field of Sports Medicine has focused on post injury concepts such as treatment, rehabilitation, and evaluation. These studies, while functioning to greatly improve the care of injured individuals, do not typically address the underlying causes of athletic injury. Thus, the recent increase in research within the field, while improving treatment of athletic injuries has not been able to effectively bridge the gap between the disciplines of Sports Medicine and Applied Sports Science.

The field of Applied Sports Science more often works from a prospective approach in an effort to identify factors capable of enhancing performance and preventing injury. As with Sports Medicine research, the field of Applied Sports Science has seen a dramatic growth in the research being conducted in recent years. This is evident from the rise of sport performance and injury prevention centers across the globe, as well as the increase in the number of Sport Science Journals being published. Unfortunately, although research in both the Applied Sports Science and Sports Medicine fields has increased dramatically, access to this research remains problematic. This arises from the fact that most research is conducted in academic settings and published in Journals and/or databases where access is limited. The development of this paradigm is dangerous as it is thought that both research and education are core factors for determining the success of sports medicine professionals [1]. Although the magnitude of research in these fields has increased, access to research findings remains limited. Thus it is the inadequacy of communication between researcher and practitioner that prevents Sports Medicine professionals from staying at the forefront of their field.

To address this issue of research availability, open access journals and databases have been increasing in number. These open access portals allow the dissemination of research findings to any and all users, free of charge. One particular publication that facilitates this communication between researchers and Sports Medicine professionals is the open access Journal of Sports Medicine and Doping Studies [2] published by the OMICS group [3]. This particular Journal, as well as similar publications maintained by other groups function to increase the availability of novel research to Sports Medicine professionals. As a result, communication between applied researchers and practicing sports Medicine professional has begun to increase. This increase in communication, if continued, may provide Sports Medicine professionals with the evidentiary support necessary for those they assist to receive the best possible assistance.

Additionally, if we are to focus on continued communication as the key factor in successfully addressing gaps within the Sports Medicine profession, we must not only continue expanding access to scientific literature through open access journals and databases, we must also begin utilizing the additional areas available for open communication between the researcher and clinician. These areas include open access digital books, audio versions and their accurate translations of publications into a variety of languages, and social networking opportunities. By focusing on these areas, we can begin to build support teams for the Sports Medicine professional in which access to scientific research is freely available, constructive communication between the researcher and practitioner is fostered, and debate addressing current issues is encouraged by each individual involved.

As evidenced, members of the Medical, Sports Medicine and Sports Science communities have worked diligently to increase the research being conducted. Although this has been done in an effort to provide the best possible care, the lack of access to this research may be preventing adequate care. Thus, now we must work to shift our focus towards enhancing the availability of research to the practicing Sports Medicine professional so we can facilitate their ability to remain at the forefront of their profession. Although this task may seem daunting, the growth and development of the open access publications and technological growth that has provided an opportunity for open and free communication are two readily available avenues through which functioning partnerships can be forged between applied sports science and sports medicine.

\section{References}

1. Whiteside J, Andrews JR (2007) Trends for the future as a team physician: Herodicus to hereafter. Clin Sports Med 26: 285-304.

2. http://omicsonline.org/jsmdshome.php

3. http://www.omicsonline.org

Corresponding author: David W. Keeley, PhD, Department of Human Performance, Dance, and Recreation, New Mexico State University, Las Cruces, USA, Tel: 575-646-5186; E-mail: dwk0611@msn.com

Received April 23, 2012; Accepted April 25, 2012; Published April 27, 2012

Citation: Keeley DW (2012) Sports Medicine and Applied Sport Science: Can we truly Bridge the Gap? J Sports Med Doping Stud 2:e104. doi:10.4172/21610673.1000e104

Copyright: (c) 2012 Keeley DW. This is an open-access article distributed under the terms of the Creative Commons Attribution License, which permits unrestricted use, distribution, and reproduction in any medium, provided the original author and source are credited. 